\title{
Minimally Invasive Single-Site Cholecystectomy in Obese Patients: Laparoscopic vs. Robotic
}

\author{
Hyeong Seok Kim, M.D., Jin-Young Jang, M.D., Ph.D. \\ Department of Surgery and Cancer Research Institute, Seoul National University College of Medicine, Seoul, Korea
}

\begin{abstract}
Robotic single-site cholecystectomy (RSSC) has emerged as an alternative procedure to overcome the shortcomings of single-fulcrum laparoscopic cholecystectomy in terms of the ergonomics and reducing the physical and mental stress of the surgeon. It is also necessary to assess the safety of RSSC for obese patients in a world with an increasing population of obese people. RSSC took a longer operation time, but the actual dissection time was similar to that of single-fulcrum laparoscopic cholecystectomy. There was no difference in postoperative pain, except for during the immediate postoperative period, and in the length of the hospital stay. RSSC had the advantage of reducing the incidence of intraoperative iatrogenic gallbladder perforation. It is critical to prevent any intraoperative bile leakage, which is associated as a worse prognostic factor in those patients with gallbladder cancer. Additional studies with a prospective design and larger number of study subjects will be required to confirm the safety and feasibility of RSSC. Moreover, the cost benefit and safety for treating malignant lesions are also issues to be addressed. RSSC is expected to be further expanded as an operative mode in the future.
\end{abstract}

Keywords: Obesity, Cholecystectomy, Laparoscopy, Robotic surgical procedures, Safety

This is an Open Access article distributed under the terms of the Creative Commons Attribution Non-Commercial License (http:// creativecommons.org/licenses/by-nc/4.0/) which permits unrestricted non-commercial use, distribution, and reproduction in any medium, provided the original work is properly cited.
Received August 7, 2019

Accepted August 16, 2019

Corresponding author

Jin-Young Jang

Department of Surgery, Seoul

National University College of

Medicine, 101 Daehak-ro,

Jongro-gu, Seoul 03080, Korea

Tel: +82-2-2072-2194

Fax: +82-2-766-3975

E-mail: jangjy4@snu.ac.kr

ORCID:

https://orcid.org/0000-0003-3312-0503
Copyright (C) 2019 The Journal of Minimally Invasive Surgery. All rights reserved.
Laparoscopic cholecystectomy (LC) is the treatment of choice for benign gallbladder (GB) disease such as GB stone, polyp and adenomyomatosis, and the area for this form of treatment has extended to early GB cancer., ${ }^{1,2}$ Many surgeons have attempted to reduce the number of ports for the LC, and single-fulcrum laparoscopic cholecystectomy (SFLC) has recently been widely performed. ${ }^{3}$ However, SFLC is associated with more physical and mental stress than that of conventional LC in terms of the ergonomics because of the limited range of motion that is due to the small size of the single-port incision. Therefore, single-port robotic surgery has emerged as an alternative to overcome the shortcomings of SFLC such as the increased surgical workload. ${ }^{5,6}$ In addition, due to the worldwide growth of the obese population, the number of surgery cases with a high body mass index (BMI) has increased compared with that in the past, and so it is necessary to assess the safety of robotic surgery in high BMI patients.

This study compared the perioperative outcomes of robotic single-site cholecystectomy (RSSC) and SFLC in high BMI $\left(>25 \mathrm{~kg} / \mathrm{m}^{2}\right)$ patients and the results showed the technical convenience and efficiency of the RSSC. The operation time was longer in the RSSC cases, but there was no difference in the actual dissection time. The immediate postoperative pain outcome was worse for RSSC, yet there was no difference in pain at the time of discharge and for the length of the hospital stay. It is noteworthy that there was significantly less intraoperative iatrogenic GB perforation in the RSSC group than that in the SFLC group.

Given the difficulty of making the preoperative diagnosis of early GB cancer because of the anatomical characteristics of 
Hyeong Seok Kim and Jin-Young Jang

the GB and despite the advancement of imaging technology, it is important to prevent intraoperative iatrogenic bile leakage that will surely lead to cancer dissemination, and this is strongly associated with a worse prognosis that will negatively affect the survival of patients. Based on these results, this paper reports that RSSC will be a safe and feasible alternative for treating GB disease. As for the results of pain, pain is an individual subjective feeling, and so it is difficult to compare among patients. There is controversy over the outcomes of postoperative pain between RSSC and SFLC: thus, further research is required with using more objective indicators. With the development of the surgical skills and instruments needed for robotic surgery, the robotic platform will be more and more applied to GB malignancy as well as GB benign lesion.? Recent reports have shown comparable oncologic outcome even in those patients with advanced GB cancer. ${ }^{8}$ We anticipate the increased role of robotic surgery in the management of GB diseases in the near future.

However, the issues on cost benefit of RSSC should be considered. Currently, the medical cost of robotic surgery is $2 \sim 3$ times higher than that of open surgery. These costs will be decreased, including the costs of the equipment, the instruments and the equipment maintenance, through the development of this new technology and the competition between robotic companies in the near future.

This study is retrospective in design with only a small number of study subjects, and there is the possibility of a selection bias. However, it is expected that a collection of small retrospective studies such as this study will provide the basis for conducting further larger studies, and all this will lead to good evidence supporting the safety and feasibility of RSSC for performing gallbladder surgery.

\section{ORCID}

Hyeong Seok Kim, https://orcid.org/0000-0003-4711-3603

Jin-Young Jang, https://orcid.org/0000-0003-3312-0503

\section{AUTHORS' CONTRIBUTIONS}

Conceptualization: JYJ. Formal analysis: HSK, JYJ. Methodology: HSK, JYJ. Writing-original draft: HSK. Writingreview and editing: HSK, JYJ.

\section{CONFLICT OF INTEREST}

None.

\section{FUNDING}

None.

\section{ACKNOWLEDGMENTS}

None.

\section{REFERENCES}

1) Begos DG, Modlin IM. Laparoscopic cholecystectomy: from gimmick to gold standard. J Clin Gastroenterol 1994;19:325-330.

2) Kim HS, Park JW, Kim H, et al. Optimal surgical treatment in patients with T1b gallbladder cancer: An international multicenter study. J Hepatobiliary Pancreat Sci 2018;25:533-543.

3) Jorgensen LN, Rosenberg J, Al-Tayar H, Assaadzadeh S, Helgstrand F, Bisgaard T. Randomized clinical trial of singleversus multi-incision laparoscopic cholecystectomy. Br J Surg 2014;101:347-355.

4) Scerbo MW, Britt RC, Stefanidis D. Differences in mental workload between traditional and single-incision laparoscopic procedures measured with a secondary task. Am J Surg 2017;213:244248.

5) Kim HS, Han Y, Kang JS, et al. Comparison of Single-Incision Robotic Cholecystectomy, Single-Incision Laparoscopic Cholecystectomy and 3-Port Laparoscopic Cholecystectomy - Postoperative Pain, Cosmetic Outcome and Surgeon\&amp;rsquo;s Workload. J Minim Invasive Surg 2018;21:168-176.

6) Grochola LF, Soll C, Zehnder A, Wyss R, Herzog P, Breitenstein S. Robot-assisted versus laparoscopic single-incision cholecystectomy: results of a randomized controlled trial. Surg Endosc 2019;33:1482-1490.

7) Shen BY, Zhan Q, Deng XX, et al. Radical resection of gallbladder cancer: could it be robotic? Surg Endosc 2012;26:3245-3250.

8) Goel M, Khobragade K, Patkar S, Kanetkar A, Kurunkar S. Robotic surgery for gallbladder cancer: Operative technique and early outcomes. J Surg Oncol 2019;119:958-963. 